\title{
Demonstration of Single-Pass Millimeterwave SAR Tomography for Forest Volumes
}

\author{
Michael Schmitt, Member, IEEE, and Xiao Xiang Zhu, Senior Member, IEEE
}

\begin{abstract}
In this letter, for the first time, the potential of millimeterwave synthetic aperture radar (SAR) is investigated with respect to a tomographic analysis of forest volumes. Exploiting both parametric and nonparametric SAR tomography (TomoSAR) methods designed for both discrete and continuous reflectivity profiles, it is shown that even Ka-band signals with a wavelength of only $8.55 \mathrm{~mm}$ can penetrate the tree canopy to a certain extent and allow a separation of ground and tree crowns. First experimental results exploiting airborne multiantenna data are evaluated with respect to LiDAR ground truth and indicate a promising perspective.
\end{abstract}

Index Terms-Forested areas, multibaseline, synthetic aperture radar (SAR) tomography, volume tomography.

\section{INTRODUCTION}

$\mathbf{S}$ INCE the first practical demonstration of synthetic aperture radar (SAR) tomography (TomoSAR), the volumetric analysis of forested areas by this technique has been an important research topic [1]. In this context, most of the literature has focused on long-wavelength radar, such as L- or P-band [2]-[4]. Only few experiments have investigated the potential of shorter wavelength SAR using X-band sensors [5]. However, recently, a TomoSAR inversion method aiming at the reconstruction of discrete scattering profiles [6] has been proposed, which has already been used to generate detailed 3-D point clouds of forested areas using Ka-band data with a wavelength in the millimeterwave domain [7]. Based on these point clouds, even the 3-D reconstruction of individual trees could be demonstrated [8]. The results of these studies indicated that millimeterwave SAR provides the advantage of showing almost no canopy penetration and therefore providing accurate height estimates almost comparable to LiDAR remote sensing. In contrast, it is still an open question whether millimeterwave signals do provide any canopy penetration at all, and whether they could potentially be employed for a TomoSAR analysis of the whole forest volume. This letter provides the firstever demonstration of volume SAR tomography using airborne multiantenna millimeterwave SAR data.

Manuscript received August 7, 2015; revised October 13, 2015 and November 17, 2015; accepted December 2, 2015. This work was supported by the Helmholtz Association under the framework of the Young Investigators Group "SiPEO" (VH-NG-1018, www.sipeo.bgu.tum.de).

The authors are with Signal Processing in Earth Observation, Technical University of Munich, 80333 Munich, Germany, and also with the Remote Sensing Technology Institute, German Aerospace Center (DLR), 82234 Wessling, Germany (e-mail: m.schmitt@tum.de; xiao.zhu@dlr.de).

Color versions of one or more of the figures in this paper are available online at http://ieeexplore.ieee.org.

Digital Object Identifier 10.1109/LGRS.2015.2506150

\section{Millimeterwave SAR Characteristics}

Since millimeterwave SAR systems are not as common in the remote sensing community as, e.g., X- or L-band systems, the characteristics of this particular microwave domain are shortly sketched here. In this context, a German experimental airborne millimeterwave interferometric SAR (InSAR) sensor is used for demonstration. The peculiarities of millimeterwave SAR have already been discussed in, e.g., [9] or [10]. However, a short recapitulation with respect to very high resolution InSAR and TomoSAR applications certainly is within the scope of this letter.

\section{A. Some Millimeterwave Peculiarities}

Typical wavelengths of millimeterwave frequencies differ from the common radar bands (L, C, X) in about one order of magnitude. This leads to two main advantages of millimeterwave systems. First, they enable a significant miniaturization of the sensor hardware, which makes them particularly feasible for use on unmanned aerial vehicles (UAVs). Second, it is possible to achieve very high resolutions with comparably short synthetic apertures. This eventually means that images of vegetated areas can be well focused because the blurring effect caused by wind-induced movements of leaves, branches, etc., is reduced. This already provides a significant benefit, when it comes to a detailed analysis of forested areas aiming at the single-tree level.

Concerning the signal propagation through the atmosphere, already Skolnik [11] has discussed that millimeterwaves can provide an interesting alternative to X-band sensors. For example, Danklmayer and Chandra [12] have shown that Ka-band imaging capabilities are available more than $95 \%$ of the time, even in rain-prone regions of the world, although attenuation caused by precipitation is, of course, significantly less for longer wavelength radars.

In general, rough surfaces cause diffuse scattering, whereas smooth surfaces result in specular reflections. At millimeterwave frequencies, most surfaces appear rough, and diffuse scattering dominates the images, leading to coherent averaging within the resolution cells. Since this is an effect similar to multilook processing, the inherent speckle effect appears less severe than in common radar bands. In addition, the high sensitivity with respect to surface roughness certainly provides a benefit, when analysis techniques based on distributed scatterers rather than point scatterers are used.

In the context of InSAR processing, one of the main differences, with respect to longer wavelengths, is the different amount of volume penetration. While L- or P-band SAR is expected to penetrate most (vegetation) volumes down to the 
TABLE I

PARAMETERS OF THE MEMPHIS TEST DATA

\begin{tabular}{ll}
\hline System parameters & \\
Carrier frequency & $35 \mathrm{GHz}$ \\
Wavelength & $8.543 \mathrm{~mm}$ \\
Acquisition parameters & \\
Flight heading angle & $200^{\circ}$ \\
Flying altitude & $760 \mathrm{~m}$ \\
Off-nadir angle & $56^{\circ}$ \\
$\quad$ Slant range & $1350 \mathrm{~m}$ \\
Resolution & \\
Azimuth & $8.2 \mathrm{~cm}$ \\
Range & $16.7 \mathrm{~cm}$ \\
Pixel spacing & \\
$\quad$ Azimuth & $5.2 \mathrm{~cm}$ \\
Range & $16.7 \mathrm{~cm}$ \\
\hline
\end{tabular}

ground, the $\mathrm{X}$ - or C-band is usually expected to exhibit phase centers somewhere within the volume. In the millimeterwave domain (Ka-band to W-band), in contrast, canopy penetration is expected to be much less likely. For tomographic inversion, however, the question arises whether millimeterwaves can be used for volume analysis at all. A first answer to this question based on real-data experiments is the main scope of this letter.

\section{B. Experimental System Description}

For the demonstrations in this letter, data acquired during a 2013 campaign of the German experimental sensor MEMPHIS is analyzed. The sensor was developed by the Fraunhofer Institute for High Frequency Physics and Radar Techniques (FHR) in 1998 [13]. Although it can operate both in the Ka-band $(35 \mathrm{GHz})$ and in the W-band $(94 \mathrm{GHz})$ and offers a fully polarimetric configuration, for the investigations presented in this letter, only the $\mathrm{HH}$ data of the Ka-band system are considered. In this interferometric configuration, MEMPHIS provides four receiving antennas, thus being a multibaseline sensor with an overall baseline span (or elevation aperture) of $27.5 \mathrm{~cm}$, which leads to a Rayleigh resolution of $\rho_{\eta} \approx 42 \mathrm{~m}$ in the elevation direction. The relevant system parameters can be found in Table I.

\section{Single-PASS MillimeterwaVe SAR TOMOgRAPHY}

\section{A. TomoSAR Inversion for Discrete and Continuous Reflectivity Profiles}

For the sake of simplicity, two of the best known and most simple to implement spectral analysis techniques are used for the evaluation of the millimeterwave test data. MUltiple SIgnal Classification (MUSIC) is a parametric spatial frequency estimator for signals affected by white noise with superresolution capabilities [14]. It is specifically designed for point scatterers, i.e., for discrete scattering profiles. Being a subspace-based technique, it aims at separation of the signal and noise subspaces by eigendecomposition of the covariance matrix of the investigated resolution cell, as follows:

$$
\mathbf{C}=\left[\begin{array}{ll}
\mathbf{E}_{s} & \mathbf{E}_{n}
\end{array}\right]\left[\begin{array}{cc}
\mathbf{D}_{s} & \mathbf{0} \\
\mathbf{0} & \mathbf{D}_{n}
\end{array}\right]\left[\begin{array}{l}
\mathbf{E}_{s} \\
\mathbf{E}_{n}
\end{array}\right]
$$

where $\mathbf{D}=\left[\begin{array}{cc}\mathbf{D}_{s} & \mathbf{0} \\ \mathbf{0} & \mathbf{D}_{n}\end{array}\right]$ is the matrix containing the eigenvalues of $\mathbf{C}$ in descending order, and $\mathbf{E}=\left[\begin{array}{ll}\mathbf{E}_{s} & \mathbf{E}_{n}\end{array}\right]$ is the matrix

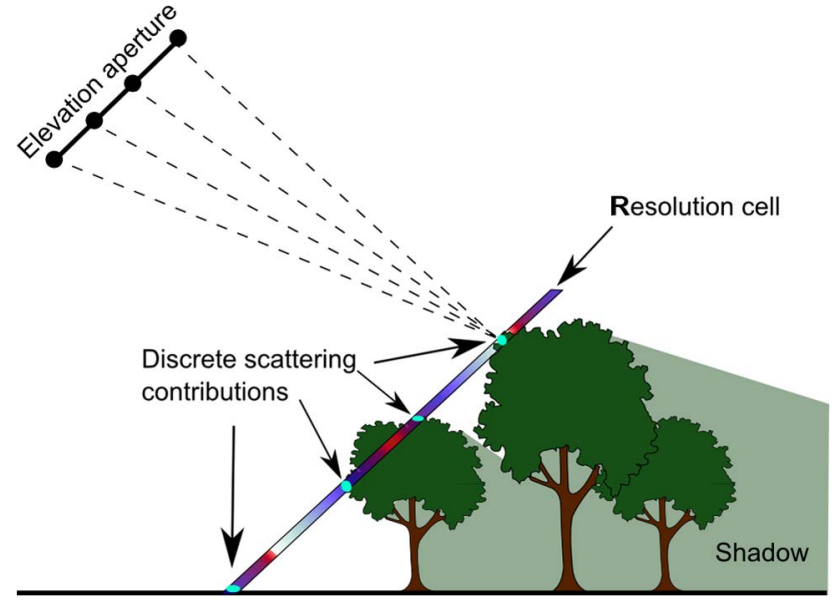

Fig. 1. Sketch of the TomoSAR acquisition geometry for forested areas. If the continuous reflectivity hypothesis is used, the whole reflectivity profile of the resolution cell is reconstructed and will probably show stronger reflectivities at or inside tree structures. In contrast, if the discrete reflectivity hypothesis is used, then only discrete scattering contributions at the sensor-facing tree structures are expected.

containing the corresponding eigenvectors. For TomoSAR inversion, then, the so-called MUSIC pseudospectrum

$$
P_{\operatorname{MusiC}}(\eta)=\frac{1}{\mathbf{a}^{H}(\eta) \mathbf{E}_{n}^{H} \mathbf{E}_{n} \mathbf{a}(\eta)}
$$

is calculated, where $\mathbf{a}(\eta)$ is the steering vector corresponding to a scattering contribution expected at elevation $\eta$. Although only the noise-related eigenvectors are used for spectral analysis, it has been shown that MUSIC exhibits significant superresolution capabilities and generally provides better results than classic beamforming or adaptive beamforming using Capon's method [15].

Nevertheless, the Capon estimator [16] is used as an example for a nonparametric estimator aiming at continuous reflectivity profiles. In contrast to MUSIC, it is not based on eigenvector analysis. Instead, it uses the inverse of the covariance matrix, in order to weight the individual elevations adaptively according to their estimated power. The Capon spectrum therefore is simply calculated by

$$
P_{\mathrm{CAPON}}(\eta)=\frac{1}{\mathbf{a}^{H}(\eta) \mathbf{C}^{-1} \mathbf{a}(\eta)}
$$

A schematic sketch of the TomoSAR configuration for forested areas, comparing both the continuous and the discrete reflectivity hypotheses, is presented in Fig. 1. In the remainder of this letter, the amplitudes of the (pseudo)reflectivities are shown in decibels, for display purposes, as follows:

$$
\tilde{P}(\eta)[\mathrm{dB}]=10 \cdot \log _{10}|P(\eta)|
$$

\section{B. Test Data}

The available experimental MEMPHIS data were acquired during a campaign over Munich, Germany, in 2013. The test scene contains the "Alter Nordfriedhof," an abandoned 


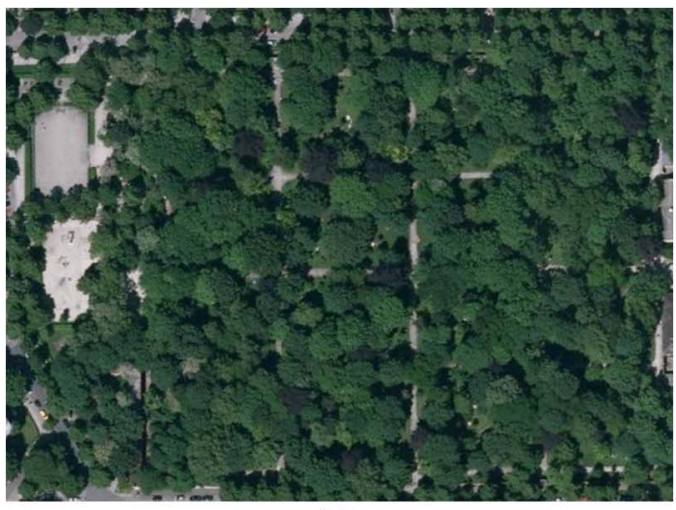

(a)

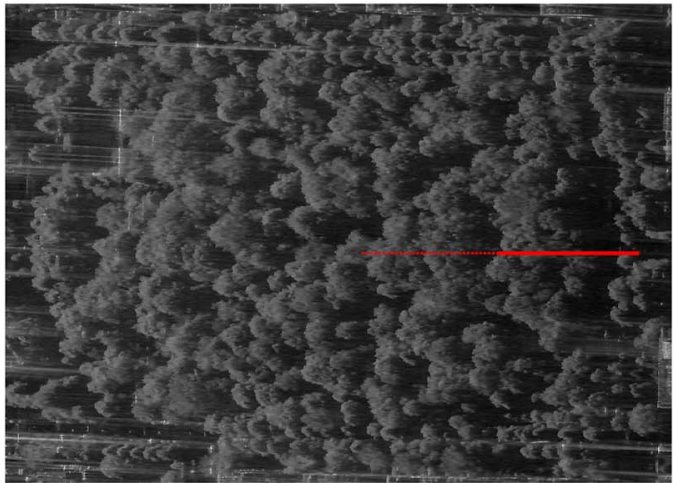

(b)

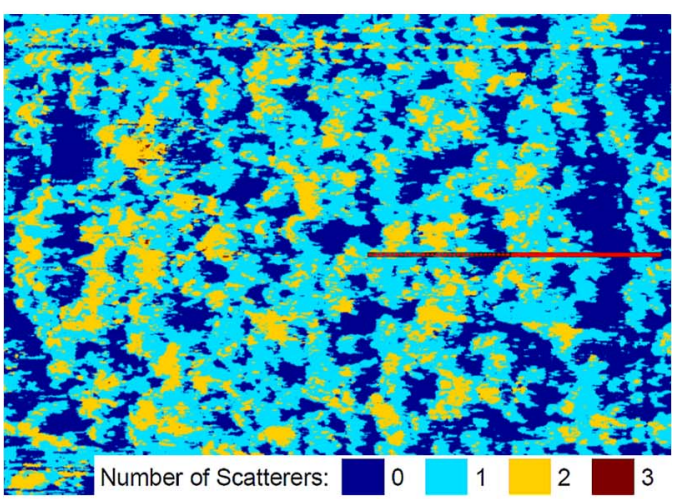

(c)

Fig. 2. Test scene. (a) Optical image. (b) SAR intensity image (SAR range direction from left to right). (c) Model order map. The red marking indicates the test profile used in the experimental section: the solid line indicates the tomograms shown in Fig. 3, whereas the dashed line indicates the extension displayed in Fig. 5.

cemetery, which is used as a public park today. As shown in Fig. 2(a), it is mainly characterized by a light planting of trees, resembling a grove or little wood. A corresponding SAR intensity image is shown in Fig. 2(b). The model order map displayed in Fig. 2(c) was calculated by the method described in [6]. Details about the test data are listed in Table I.

The ground truth used for the following evaluations was acquired during a terrestrial laser scanning (TLS) campaign in July 2014. From three scanning locations, three point clouds with approximately 35 million points each (point density approximately $2500 \mathrm{pts} / \mathrm{m}^{2}$ ) were created and coregistered afterward. Details about the resulting data set are summarized in Table II.
TABLE II

TECHNICAL SPECIFICATIONS OF THE TLS USED FOR GROUND TRUTH GENERATION

\begin{tabular}{ll}
\hline System & Leica HDS700 \\
\hline Scanning type & Phase based \\
Wavelength & $1.5 \mu \mathrm{m}$ \\
Range & $0.3 \mathrm{~m} . .187 \mathrm{~m}$ \\
Scan resolution & $0.6 \mathrm{~mm} / 10 \mathrm{~m}$ \\
Angular resolution & $7 \mu \mathrm{rad}$ \\
Angular accuracy & $125 \mu \mathrm{rad}$ \\
Linearity error & $\leq 1 \mathrm{~mm}$ \\
\hline
\end{tabular}

\section{Experimental Results}

Fig. 3 shows the tomographic slices corresponding to the solid line in Fig. 2, which were processed with both Capon and MUSIC for different model orders. In addition, the LiDAR ground truth projected into radar geometry (green points) is shown for comparison in Fig. 4. While Fig. 3(a) shows the classical nonparametric Capon tomogram, Fig. 3(b)-(d) shows the MUSIC tomograms for fixed model orders of $K=\{1,2,3\}$ (i.e., $K$ eigenvectors of each resolution cell's sample covariance matrix are used for spanning the signal subspace and $4-K$ for spanning the noise subspace).

In order to provide more material for further analysis, additionally, a MUSIC tomogram with automatic model order selection [corresponding to the model order map shown in Fig. 2(c)] is provided in Fig. 5. It is extended by some range bins toward the sensor (marked by the dashed line in Fig. 2), discretized, and geocoded as point cloud, with the points being colorized by the pseudointensities of the respective range-elevation cell. This way, the tomographic data can be overlayed with the LiDAR ground truth in world geometry.

In all tomograms, an overall agreement between the forest structure and the tomogram reflectivities can be seen, whereas Fig. 5 also contains the shadowed resolution cells, where no scattering contribution (or no signal-related eigenvalueeigenvector pair, that is) was detected. From these results, three interesting features can be discovered:

- There are scene parts, which should actually be covered by tree canopy, but are still part of the tomographic reconstruction (as an example, see rectangle in Fig. 5).

- Although MUSIC pseudoreflectivities and Capon reflectivities are not directly related, in both results the strongest values are found at the tree crowns facing the sensor. In addition, strong (pseudo)reflectivities are also found within the tree crowns and, for superresolving MUSIC, at the approximate ground level.

- While the model order map in Fig. 2(c) generally looks quite reasonable with seemingly proper shadow detection and a large part of the scene affected by layover $(K=2)$, higher model orders obviously contain more information, which should not be neglected.

All three phenomena indicate that there needs to be a certain penetration of the canopy by the millimeterwave signals. Particularly noteworthy is the situation at the marked gravestone located in the center of the scene (cf. Fig. 5): Here, quite strong scattering occurs, although the monument is actually in the radar shadow of several trees and bushes in front of it. Obviously, a combination of low leaf density and strong backscattering of the man-made object allows for a certain amount of subcanopy imaging in this case. 


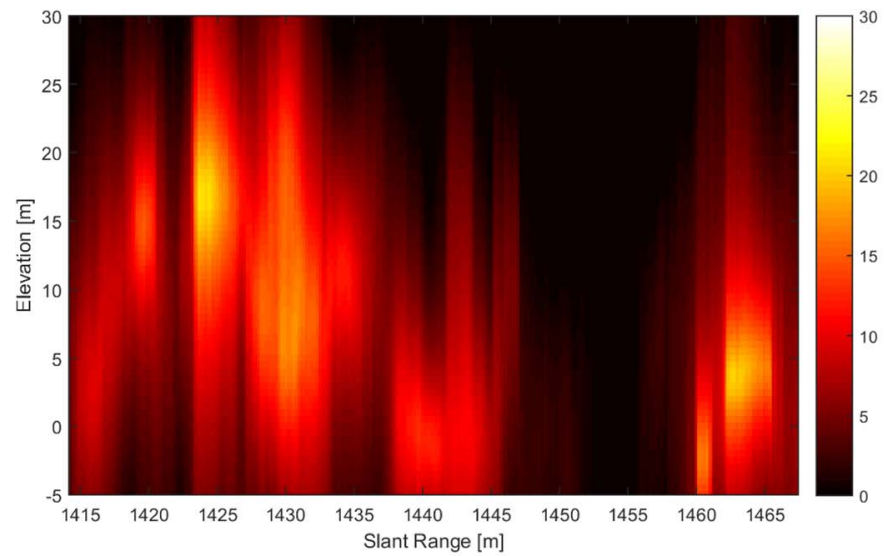

(a)

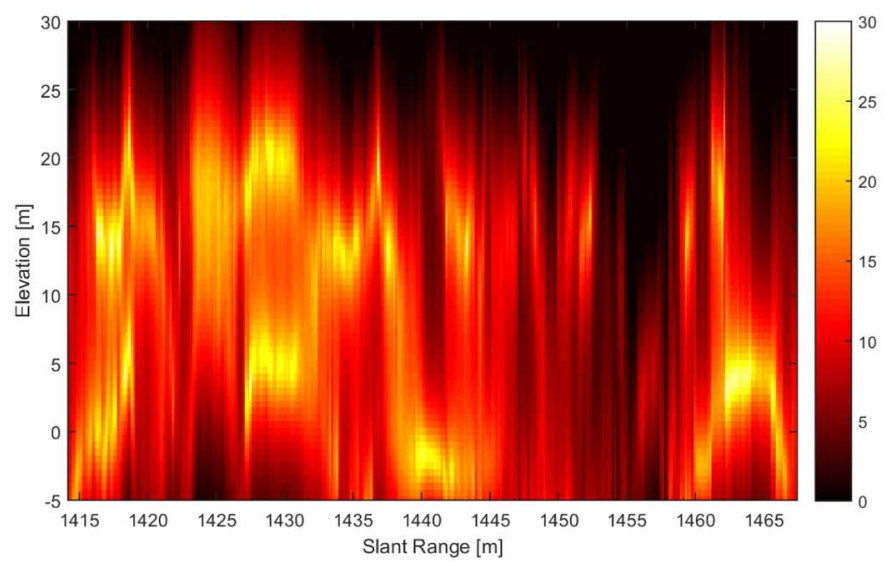

(c)

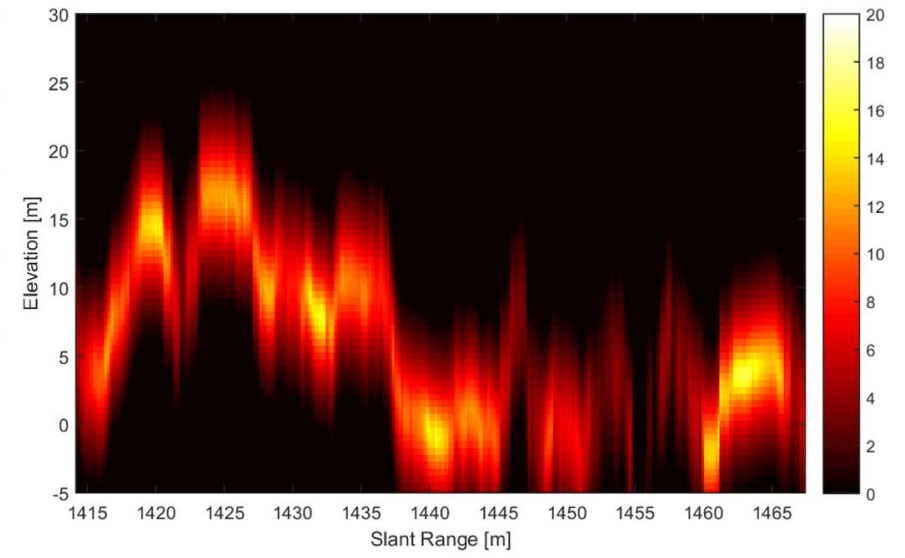

(b)

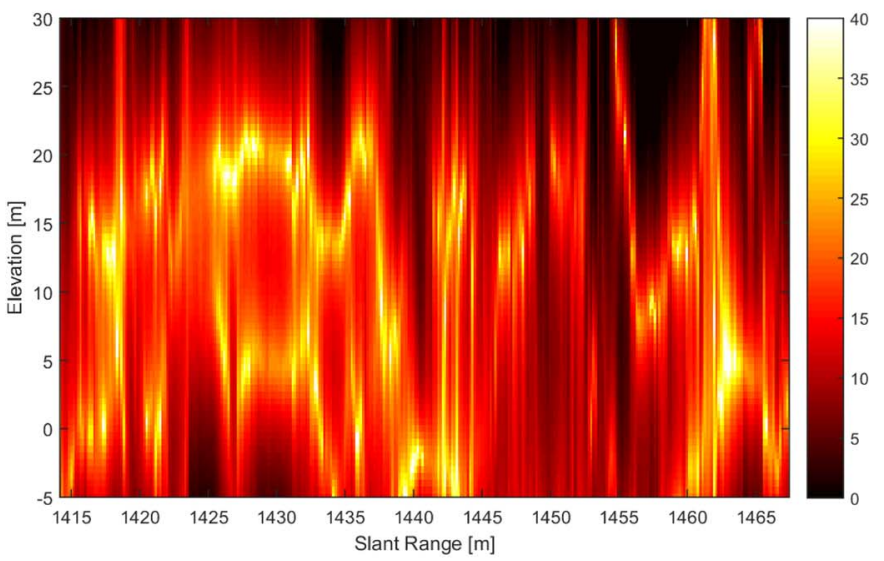

(d)

Fig. 3. (a) Capon tomogram. MUSIC tomograms with (b) $K=1$, (c) $K=2$, and (d) $K=3$. The (pseudo)intensities [in decibels] range from red (low) to yellow (high).

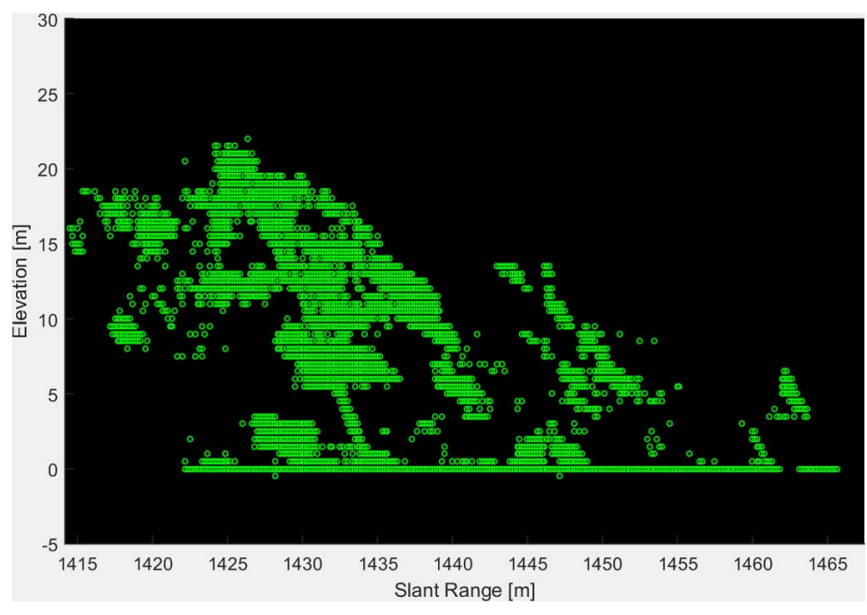

Fig. 4. LiDAR ground truth projected in the slant range-elevation plane of the radar geometry.

\section{Discussion}

The experimental results summarized in Section III-C show that there is indeed a certain amount of canopy penetration for millimeterwave SAR signals. Using MUSIC-based inversion in conjunction with a continuous TomoSAR model, strong pseudoreflectivities not only within tree crowns but also in underfoliage scene parts, where radar shadowing from higher trees would be expected, can be seen. As the in situ photograph displayed in Fig. 6 shows, this is probably related to the low leaf density in the tree canopy. In addition, it seems that subcanopy backscattering is supported by strong reflections provided by man-made structures made of concrete.

In addition, it has to be noted that wrong model order selection can lead to a severe underestimation of the amount of present signal information. Looking, e.g., at the range bins 1425 to 1435 , strong backscattering from both the tree canopy and the undergrowth for $K=2$ and $K=3$ are revealed, while Capon and MUSIC with $K=1$ only show a weak response at intermediate elevation. Particularly for single-pass systems with a very low number of available acquisitions $(N=4$ in the MEMPHIS case), the relevant signal content of volumetric media is spread throughout the few available eigenvalues, such that it is advisable to always choose $K$, as high as possible, in order to retrieve as much information as possible.

\section{Summary AND CONCLUSION}

In this letter, the very first experimental results for millimeterwave SAR tomography of forest volumes have been shown. Using test data acquired by the single-pass multibaseline interferometer MEMPHIS and a ground truth data set acquired by high-precision TLS, it could be shown that there is a certain amount of canopy penetration, if the leaf density is not too high. 


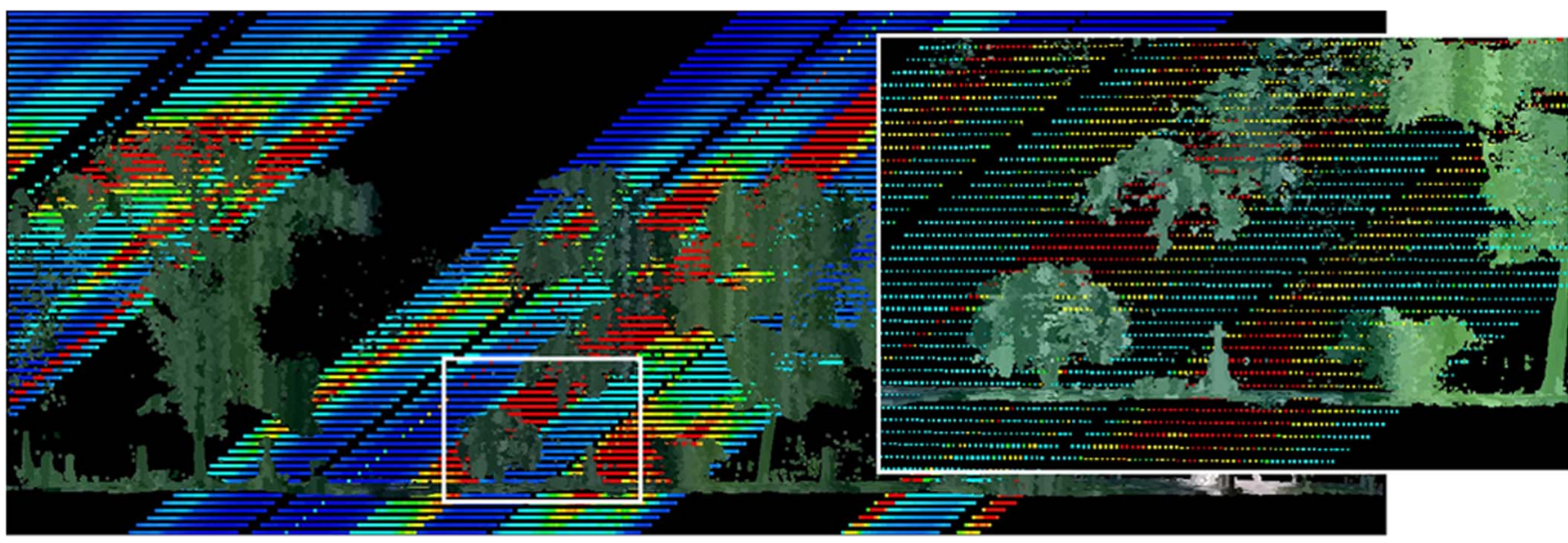

Fig. 5. Geocoded MUSIC tomogram with automatic model order selection overlayed to the TLS point cloud in world geometry. SAR viewing direction is from the upper left. The (pseudo)intensities range from blue (low) to red (high), and the white box indicates the zoomed detail view on the right.

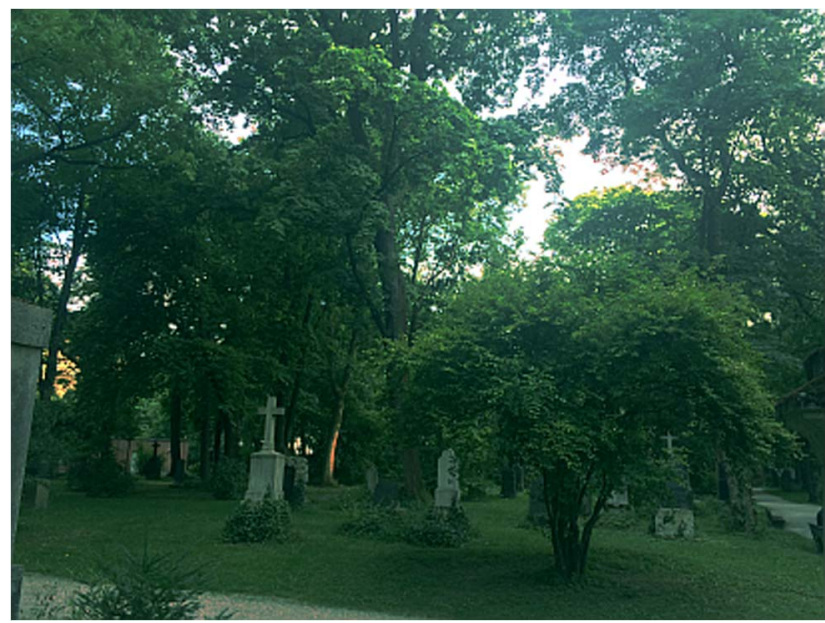

Fig. 6. In situ photograph of the test area showing the gravestone and an exemplary section of the canopy.

This asks for more investigations with respect to subcanopy target detection, particularly if the object of interest provides strong enough backscattering. However, the main reflectivity contributions occur at the tree crowns facing the sensor. Therefore, also a discrete scattering model can be employed for reconstruction aiming at the canopy only, making this model a promising perspective for canopy height model generation or even individual tree reconstruction, as proposed in [8].

\section{ACKNOWLEDGMENT}

The authors would like to thank T. Brehm and Dr. S. Stanko from the Fraunhofer Institute for High Frequency Physics and Radar Techniques (FHR), for providing the MEMPHIS test data; C. Magnard and Dr. E. Meier from the Remote Sensing Laboratories at the University of Zurich, for focusing the raw SAR data; and geodesists P. Schreiner and W. Wiedemann from the Technical University of Munich, for the efforts concerning the acquisition and processing of the LiDAR ground truth data.

\section{REFERENCES}

[1] A. Reigber and A. Moreira, "First demonstration of airborne SAR tomography using multibaseline L-band data," IEEE Trans. Geosci. Remote Sens., vol. 38, no. 5, pp. 2142-2152, Sep. 2000.

[2] O. Frey, F. Morsdorf, and E. Meier, "Tomographic imaging of a forested area by airborne multi-baseline P-band SAR," Sensors, vol. 8, no. 9, pp. 5884-5896, Sep. 2007.

[3] O. Frey and E. Meier, "Analyzing tomographic SAR data of a forest with respect to frequency, polarization, and focusing technique," IEEE Trans. Geosci. Remote Sens., vol. 49, no. 10, pp. 3648-3659, Oct. 2011.

[4] S. Tebaldini and F. Rocca, "Multibaseline polarimetric SAR tomography of a boreal forest at P- and L-bands," IEEE Trans. Geosci. Remote Sens., vol. 50, no. 1, pp. 232-246, Jan. 2012.

[5] J. Praks, F. Kugler, J. Hyyppa, K. Papathanassiou, and M. Hallikainen, "SAR coherence tomography for boreal forest with aid of laser measurements," in Proc. IEEE Int. Geosci. Remote Sens. Symp., 2008, pp. 469-472.

[6] M. Schmitt and U. Stilla, "Maximum-likelihood-based approach for single-pass synthetic aperture radar tomography over urban areas," IET Radar, Sonar Navig., vol. 8, no. 9, pp. 1145-1153, Dec. 2014.

[7] M. Schmitt and U. Stilla, "Generating point clouds of forested areas from airborne millimeter wave InSAR data," in Proc. IEEE Int. Geosci. Remote Sens. Symp., Jul. 2014, pp. 1-4.

[8] M. Schmitt, M. Shahzad, and X. Zhu, "Reconstruction of individual trees from multi-aspect TomoSAR data," Remote Sens. Environ., vol. 165, pp.175-185, Aug. 2015.

[9] H. Essen, "Airborne remote sensing at millimeter wave frequencies," in Radar Remote Sensing of Urban Areas, U. Soergel, Ed. Dordrecht, The Netherlands: Springer-Verlag, 2010.

[10] M. Schmitt, C. Magnard, S. Stanko, C. Ackermann, and U. Stilla, "Advanced high resolution SAR interferometry of urban areas with airborne millimetrewave radar," Photogramm. Fernerkundung Geoinf., vol. 2013, no. 6, pp. 603-617, 2013.

[11] M. Skolnik, Introduction to Radar Systems. New York, NY, USA: McGraw-Hill, 1980.

[12] A. Danklmayer and M. Chandra, "Precipitation effects for Ka-band SAR," in Proc. Adv. RF Sensors Earth Observ., Noordwijk, The Netherlands, 2009, pp. 1-8.

[13] H. Schimpf, H. Essen, S. Boehmsdorff, and T. Brehm, "MEMPHISA fully polarimetric experimental radar," in Proc. IEEE Int. Geosci. Remote Sens. Symp., 2002, pp. 1714-1716.

[14] R. O. Schmidt, "Multiple emitter location and signal parameter estimation," IEEE Trans. Antennas Propag., vol. AP-34, no. 3, pp. 276-280, Mar. 1986.

[15] F. Gini and F. Lombardini, "Multibaseline cross-track SAR interferometry: A signal processing perspective," IEEE Aerosp. Electron. Syst. Mag., vol. 20, no. 8, pp. 71-93, Aug. 2005.

[16] J. Capon, "High-resolution frequency-wavenumber spectrum analysis," Proc. IEEE, vol. 57, no. 8, pp. 1408-1418, Aug. 1969. 